\title{
ATIVIDADE ANTIOXIDANTE E FENÓLICOS TOTAIS EM BLENDS DE SUCOS DE UVAS AMERICANAS PRODUZIDAS NO SUDOESTE DE MINAS GERAIS
}

\author{
ANA CAROLINA VILAS BOAS* \\ PAÔLA DE CASTRO HENRIQUE* \\ LUIZ CARLOS DE OLIVEIRA LIMA*** \\ ANTÔNIO DECARLOS NETO**** \\ RITA DE CÁSSIA MIRELA RESENDE NASSUR \\ RAFAELLA ARAÚJO ZAMBALDI LIMA
}

\begin{abstract}
O presente trabalho teve como objetivo a caracterização e a avaliação da atividade antioxidante de blends de sucos de uvas americanas produzidas no sudoeste de Minas Gerais. As uvas foram colhidas e sanitizadas, sendo o engaço descartado. Então, os blends Niágara x BRS Cora $(50 \%+50 \%)$, Niágara x BRS Cora x BRS Violeta $(40 \%$ $+30 \%+30 \%)$ e Isabel Precoce $x$ Niágara $x$ BRS Cora $(25 \%+25 \%$ $+50 \%$ ) foram produzidos por método artesanal por arraste de vapor, acondicionados em frascos de vidro e armazenados a $18^{\circ} \mathrm{C} \pm 2$ ${ }^{\circ} \mathrm{C}$ por 120 dias. As seguintes análises foram realizadas ao longo do armazenamento: cor $\left(\mathrm{L}^{*}\right), \mathrm{pH}$, sólidos solúveis, acidez titulável, relação sólidos solúveis/acidez titulável, antocianinas, atividade antioxidante (DPPH e $\beta$-caroteno/ácido linoleico), fenólicos totais, vitamina $\mathrm{C}$ e ácidos orgânicos. O blend Niágara x BRS Cora x BRS Violeta apresentou maior teor de antocianinas e vitamina $\mathrm{C}$, menor acidez e maior relação sólidos solúveis/acidez titulável, indicando um maior equilíbrio entre os sabores doce e ácido.
\end{abstract}

PALAVRAS-CHAVE: SUCO DE UVA; ANTOCIANINAS; VITAMINA C; ÁCIDOS ORGÂNICOS; QUALIDA$D E$.

* Farmacêutica, doutoranda em Ciência dos Alimentos, Universidade Federal de Lavras, Lavras, MG (e-mail: vbanacarolina@yahoo.com.br).

** Bióloga, doutoranda em Ciência dos Alimentos, Universidade Federal de Lavras, Lavras, MG (e-mail: paolahenrique@yahoo.com.br).

${ }^{* * *}$ Farmacêutico, professor, doutor em Ciência dos Alimentos, Universidade Federal de Lavras, Lavras, MG (e-mail: Icolima@dca.ufla.br).

**** Agrônomo, professor, doutor em Agronomia, Universidade Federal de Lavras, Lavras, MG (e-mail: adecarlos@ dag.ufla.br).

****** Agrônoma, doutora em Ciência dos Alimentos, Universidade Federal de Lavras, Lavras, MG (e-mail: ritarnassur@hotmail.com).

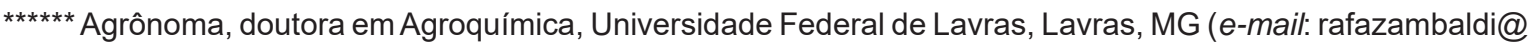
hotmail.com). 


\section{INTRODUÇÃO}

Frutas e produtos derivados do seu processamento, como sucos e vinhos, são excelentes fontes de alguns micronutrientes (minerais essenciais e vitaminas) e fitoquímicos (carotenoides e compostos fenólicos). Os polifenóis presentes nas frutas exibem atividade antioxidante considerável in vitro e representam a mais abundante fonte de antioxidantes da dieta humana (GARDNER et al., 2000).

Além disso, é sabido que o conteúdo de fenólicos do suco de uva está intimamente relacionado com as suas qualidades sensoriais, em termos de cor e adstringência (GURAK et al., 2010). Os compostos presentes na uva e seus produtos, que, presume-se, fornecem efeitos positivos à saúde, são principalmente flavonóis, procianidinas, antocianinas e ácidos fenólicos (ANDRADE et al., 2001; CHEDEA et al., 2010).

Blends são misturas de sucos, conhecidas como cortes, feitos com a finalidade de melhorar as características sensoriais e físico-químicas dos produtos elaborados, como conferir coloração a sucos de cultivares com pouca coloração, doçura, aroma e/ou sabor.

Muitos estudos têm sido realizados para determinar os compostos fenólicos e a capacidade antioxidante de sucos de uva durante o armazenamento (GENOVA et al., 2012). No entanto, ainda não estão disponíveis estudos que abordem a determinação da atividade antioxidante, compostos fenólicos, ácidos orgânicos e coloração de blends de sucos de uvas da região do sudoeste de Minas Gerais, que apresenta condições edafoclimáticas bastante diferentes daquelas apresentadas pelas tradicionais regiões vinícolas localizadas no Sul e no Nordeste do país, com clima tipicamente subtropical (LUCENA et al., 2010). Considerando a influência marcante do clima sobre as características físico-químicas da uva, é compreensível concordar que bebidas produzidas em diferentes regiões possuam características sensoriais e nutricionais bastante peculiares.

O objetivo do presente trabalho foi realizar a caracterização e a avaliação da atividade antioxidante de diferentes blends de sucos de uvas produzidas no sudoeste de Minas Gerais, Brasil.

\section{MATERIAL E MÉTODOS}

\subsection{PRODUÇÃO DOS SUCOS}

Os blends de uva foram elaborados experimentalmente no Laboratório de Enologia da Universidade Federal de Lavras (Lavras, MG, Brasil), empregando uvas da safra de 2012/2013, cultivadas na mesma região do sudoeste de Minas Gerais e provenientes do pomar institucional. Após a colheita, as uvas foram mantidas em câmara fria a $10^{\circ} \mathrm{C} \pm 2{ }^{\circ} \mathrm{C}$ durante 12 horas. Então, as frutas foram sanitizadas com hipoclorito de sódio a $200 \mathrm{mg} \mathrm{L}^{-1} \mathrm{e}$ as bagas foram manualmente degranadas. A elaboração dos blends de sucos de uva foi realizada a $75^{\circ} \mathrm{C} \pm 5^{\circ} \mathrm{C}$ durante uma hora, usando um equipamento artesanal por arraste de vapor segundo Rizzon, Manfroi e Meneguzzo (1998). Três tipos de blends foram elaborados com as seguintes variedades e nas seguintes proporções: Niágara x BRS Cora $(50 \%+50 \%)$, Niágara x BRS Cora x BRS Violeta $(40 \%+30 \%+30 \%)$ e Isabel Precoce $x$ Niágara $\times$ BRS Cora $(25 \%+25 \%+50 \%)$. Para cada litro de blend de uva, foi adicionado $0,8 \mathrm{~g}$ de metabissulfito de potássio (Synth ${ }^{\circledR}$, Diadema, Brasil). Após essa etapa, os blends foram imediatamente engarrafados em frascos de vidro de $100 \mathrm{ml}$ e mantidos sob refrigeração $\left(18{ }^{\circ} \mathrm{C} \pm 2\right.$ $\left.{ }^{\circ} \mathrm{C}\right)$ até o momento das análises.

\subsection{ANÁLISES}

As seguintes análises foram realizadas nos dias 0, 15, 30, 60 e 120 de armazenamento: cor $\left(\mathrm{L}^{*}\right), \mathrm{pH}$, sólidos solúveis, acidez titulável, relação sólidos solúveis/acidez titulável, atividade antioxidante (DPPH e $\beta$-caroteno/ácido linoleico), fenólicos totais e vitamina C. Já as análises de antocianinas e ácidos orgânicos foram realizadas nos dias 0 e 120. 
Para avaliar a coloração dos blends foi utilizado o colorímetro Minolta, modelo CR 400, no sistema da Commission Internacionale de Eclairage (CIE, 1978), pesquisando-se a coordenada L*, que mede a claridade ou a luminosidade da amostra, variando entre o preto (0) e o branco (100).

A mensuração do $\mathrm{pH}$ foi feita empregando-se um ph-metro Tecnal (Tec $3 \mathrm{M}$ ) com eletrodo de vidro, conforme recomendações da Association of Official Analytical Chemists (AOAC, 2007). Os sólidos solúveis totais dos sucos foram determinados utilizando refratômetro digital ATAGO PR-100, e os resultados, expressos em porcentagem, de acordo com técnica da AOAC (2007). A acidez titulável também foi determinada por metodologia sugerida pela AOAC (2007), realizando-se titulação com solução de hidróxido de sódio $(\mathrm{NaOH})$ 0,1 mol. $\mathrm{L}^{-1}$, sendo os resultados expressos em porcentagem de ácido tartárico. Para o cálculo da relação SST/AT foi realizada a divisão do teor de sólidos solúveis totais pela acidez titulável.

Os fenólicos totais foram obtidos conforme o método colorimétrico desenvolvido por Singleton e Rossi (1965), com a utilização do reagente de Folin-Ciocalteu, em solução com concentração de $10 \%(v / v)$. O procedimento de extração envolveu etapas consecutivas de centrifugação, filtração e repouso, visando obter uma melhor extração dos compostos fenólicos, conforme descrito por Larrauri, Rupérez e Saura-Calixto (1997).

A determinação da atividade antioxidante dos sucos foi realizada pelo método de sequestro do radical DPPH (2,2-difenil-1-picril-hidrazil) por antioxidantes, segundo Rufino et al. (2007a). Para determinar a atividade antioxidante, foram empregados os extratos utilizados para determinação dos fenólicos totais, conforme sugestão de Larrauri, Rupérez e Saura-Calixto (1997). Para fins de comparação com resultados da literatura, foi calculada a porcentagem de sequestro de radicais livres (\% SRL), conforme fórmula sugerida por Duarte-Almeida et al. (2006): \% SRL = (Ac - Am) x 100/Ac, onde Ac (Abs do controle) e Am (Abs da amostra). Nesse parâmetro, valores elevados indicam uma maior capacidade antioxidante da amostra pesquisada.

A avaliação da atividade antioxidante dos sucos pelo sistema $\beta$-caroteno/ácido linoleico seguiu protocolo recomendado por Rufino (2007b). O extrato das amostras foi obtido segundo metodologia de Larrauri, Rupérez e Saura-Calixto (1997). Os resultados, por sua vez, foram expressos em porcentagem da inibição da oxidação do sistema contra a oxidação.

A análise do conteúdo total de antocianinas foi realizada seguindo-se o método do $\mathrm{pH}$ diferencial, proposto por Giusti e Wrolstad (2001), e os resultados foram expressos em mg. L-1.

A quantificação dos teores de vitamina $C$ foi feita por método colorimétrico, empregando-se 2,4 dinitrofenil-hidrazina, segundo Strohecker e Henning (1967). A leitura foi realizada a $520 \mathrm{~nm}$ em espectrofotômetro Beckman 640B, com sistema computadorizado. Os resultados foram expressos em miligramas de ácido ascórbico. $100 \mathrm{~mL}^{-1}$ de suco.

O teor de ácidos orgânicos foi realizado por cromatografia líquida de alta eficiência (CLAE) no Centro de Análises Avançadas e Biotecnologia da Universidade Federal de Lavras. Foi utilizada uma coluna SHIM-PACK SPR-H (250 mm x 7,8 mm), equipada com pré-coluna SHIM-PACK SPH-H(G) $(50 \mathrm{~mm}$ $x 7,8 \mathrm{~mm}$ ). Como fase móvel, foram utilizados os seguintes solventes: (A) solução aquosa $4 \mathrm{mM} \rho$-ácido sulfônico tolueno; e (B) solução aquosa de $16 \mathrm{mM}$ Bis-Tris, $4 \mathrm{mM} \rho$-ácido sulfônico tolueno e $100 \mu \mathrm{m}$ EDTA. O fluxo utilizado foi de $0,8 \mathrm{ml} / \mathrm{min}$, detector de condutividade elétrica (DCE-6A) à temperatura de 43 ${ }^{\circ} \mathrm{C}$ e volume de amostra injetado de $10 \mu$ l. Como pré-tratamento da amostra, nenhum outro processo foi necessário além de filtração através de um filtro de membrana (porosidade de $0,45 \mu \mathrm{m}$ ) antes da injeção.

\subsection{DELINEAMENTO EXPERIMENTAL}

O delineamento experimental utilizado foi o inteiramente casualizado (DIC) em esquema fatorial $(3 \times 5)$ composto por três blends de sucos de uva e cinco períodos de armazenamento $(0,15$, 30,60 e 120 dias), com quatro repetições. O teste de Tukey a $5 \%$ de probabilidade foi utilizado para comparar os tratamentos dentro de cada tempo. Os modelos de regressão polinomiais, utilizados para tempo de armazenamento, foram selecionados com base na significância do teste $\mathrm{F}$ de cada modelo testado e no coeficiente de determinação. 


\subsection{VALORES DE L* E ANTOCIANINAS TOTAIS}

A figura 1 apresenta os valores de $L^{*}$, e a tabela 1 , o teor de antocianinas totais dos diferentes blends de sucos de uvas produzidas no sudoeste de Minas Gerais ao longo do armazenamento refrigerado.

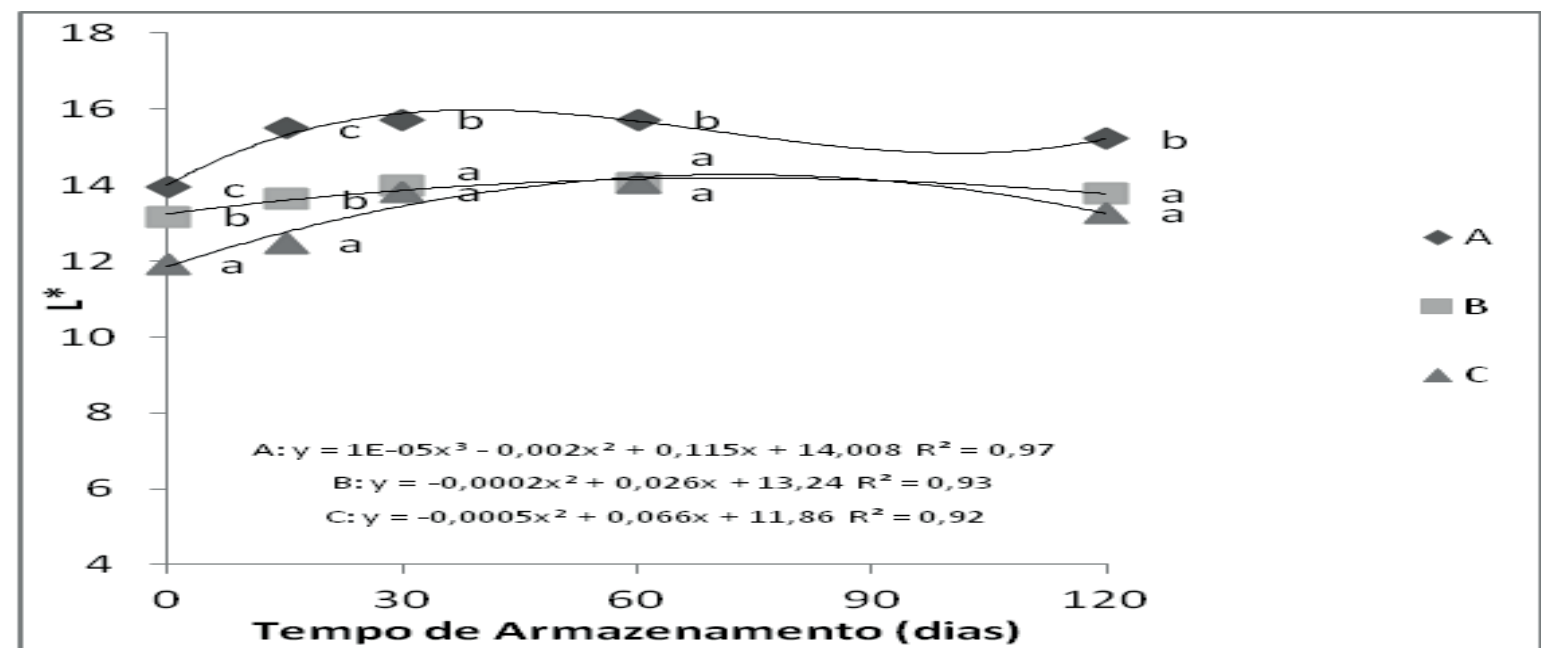

FIGURA 1 - VALORES DE L* DE DIFERENTES BLENDS DE SUCOS DE UVAS PRODUZIDAS NO SUDOESTE DE MINAS GERAIS (A: NIÁGARA ROSADA X BRS CORA,

B: ISABEL PRECOCE X NIÁGARA ROSADA X BRS CORA, C: NIÁGARA ROSADA X BRS CORA X BRS VIOLETA)

TABELA 1 - TEOR DE ANTOCIANINAS TOTAIS DE DIFERENTES BLENDS DE SUCOS DE UVAS PRODUZIDAS NO SUDOESTE DE MINAS GERAIS

\begin{tabular}{lcc}
\hline \multicolumn{1}{c}{ Antocianinas totais $\left(\mathbf{m g ~ L}^{-1}\right)$} & \\
\hline Blends & Tempo 0 & Tempo 120 \\
\hline Niágara x BRS Cora & 243,23 a A & 135,86 b A \\
\hline Isabel Precoce x Niágara x BRS Cora & 272,45 a B & 173,04 b B \\
\hline Niágara x BRS Cora x BRS Violeta & 459,84 a C & 303,62 b C \\
\hline
\end{tabular}

Os valores de $L^{*}$, que variam do preto ao branco, mostraram diferenças significativas entre os blends, sendo o Niágara x BRS Cora x BRS Violeta o que apresentou menores valores, indicando um blend mais tinto. Consequentemente aos valores L*, o blend Niágara x BRS Cora $\times$ BRS Violeta apresentou o maior conteúdo de antocianinas totais entre as amostras, sofrendo uma redução ao longo do tempo de armazenamento. Durante a estocagem podem ocorrer mudanças no aroma, na cor e no sabor do suco devido à redução na concentração de antocianinas monoméricas e à formação de pigmentos poliméricos. As reações responsáveis por essas transformações incluem, frequentemente, a condensação direta entre antocianinas e flavonóis e a polimerização das próprias antocianinas (FRANCIA-ARICHA et al., 1997).

\subsection{CARACTERIZAÇÃO FÍSICO-QUÍMICA}

As características físico-químicas dos diferentes blends de sucos de uvas produzidas no sudoeste de Minas Gerais são apresentadas na figura 2. 


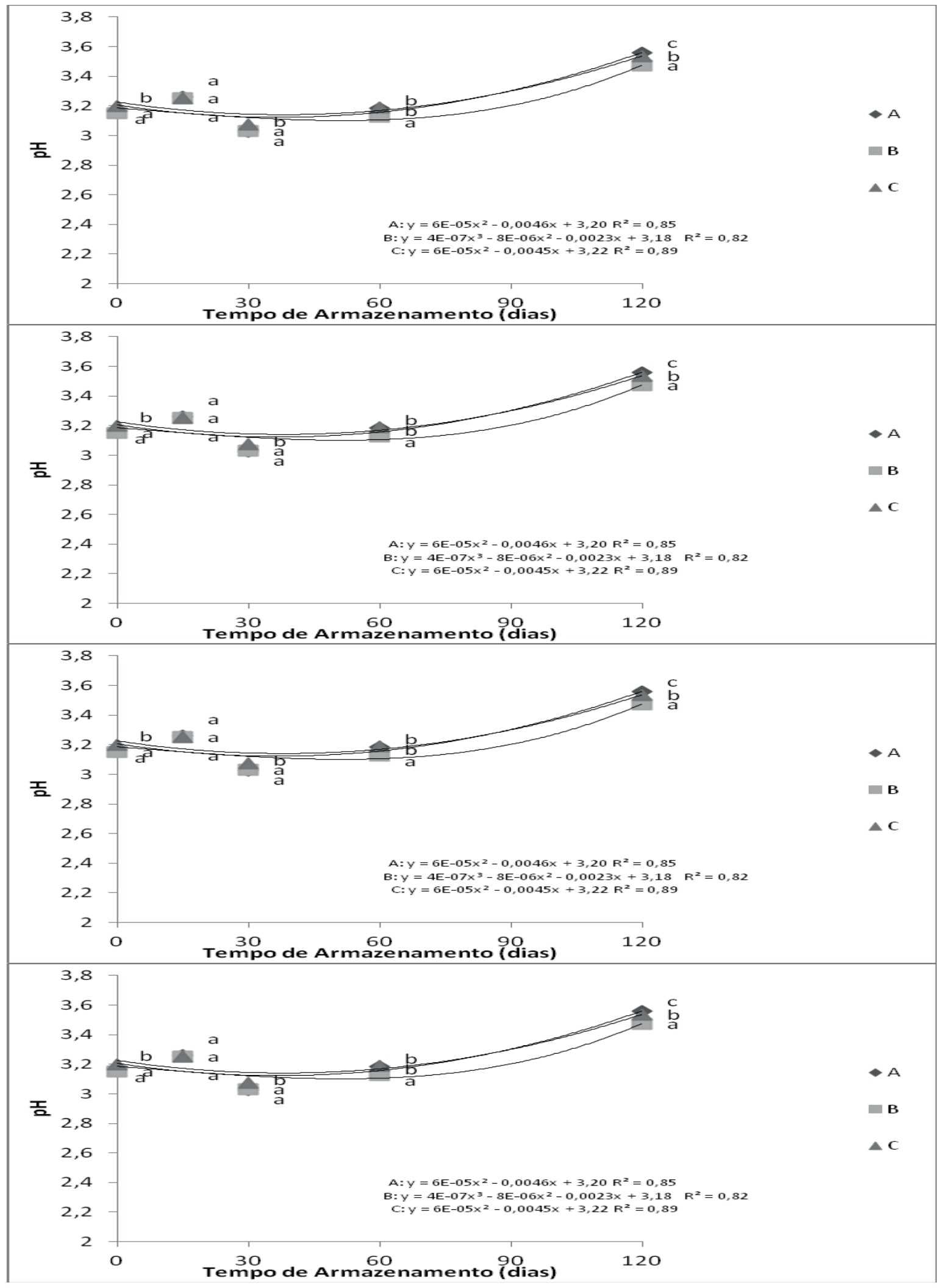

FIGURA 2 - PH, SÓLIDOS SOLÚVEIS TOTAIS, ACIDEZ TITULÁVEL E RELAÇÃO SÓLIDOS SOLÚVEIS/ACIDEZ TITULÁVEL DE DIFERENTES BLENDS DE SUCOS DE UVAS PRODUZIDAS NO SUDOESTE DE MINAS GERAIS (A: NIÁGARA ROSADA X BRS CORA, B: ISABEL PRECOCE X NIÁGARA ROSADA X BRS CORA, C: NIÁGARA ROSADA X BRS CORA X BRS VIOLETA) 
Todos os blends elaborados apresentaram um aumento de $\mathrm{pH}$ no final do período de armazenamento, atingindo valores médios de 3,24, 3,20 e 3,25 para os blends Niágara x BRS Cora, Isabel Precoce $x$ Niágara x BRS Cora e Niágara x BRS Cora x BRS Violeta, respectivamente. Borges et al. (2011), trabalhando com os blends Isabel + BRS Cora $(80 \%$ $+20 \%)$ e Isabel + BRS Violeta $(80 \%+20 \%)$, encontraram, respectivamente, valores de 3,29 e 3,30, próximos aos encontrados neste estudo. Acredita-se que os maiores valores de $\mathrm{pH}$ encontrados no final do período de armazenamento sejam consequência dos menores teores de ácidos orgânicos no referido tempo, evidenciados pela análise de acidez titulável, já que esses parâmetros estão frequentemente interligados, segundo Rizzon, Miele e Meneguzzo (2000). Assim, todos os blends apresentaram uma redução da acidez titulável, indicando que ficaram menos ácidos ao longo do tempo. No que se refere à acidez titulável, a legislação brasileira estabelece um mínimo de $0,49 \%$ de ácido tartárico (BRASIL, 1998). Sendo assim, todos os blends ficaram acima do nível mínimo estabelecido para acidez titulável, sendo o blend Niágara x BRS Cora x BRS Violeta o que apresentou menores valores, atingindo $0,82 \%$ aos 120 dias de armazenamento. Borges et al. (2011), avaliando diferentes blends de uva, encontrou resultados parecidos para acidez titulável no blend Isabel x Cora $(80 \%+20 \%)$, com valores de $0,78 \%$ de ácido tartárico.

A acidez em produtos hortícolas é atribuída, principalmente, aos ácidos orgânicos que se encontram dissolvidos nos vacúolos das células, tanto na forma livre como na combinada, como sais, ésteres, glicosídeos, etc. Em alguns produtos, os ácidos orgânicos não só contribuem para a acidez, como também para o aroma característico, porque alguns componentes são voláteis (CHITARRA e CHITARRA, 2005).

Com relação ao teor de sólidos solúveis totais, todos os blends elaborados encontraram-se abaixo do mínimo preconizado pela legislação brasileira para suco de uva, que é de $14{ }^{\circ} \mathrm{Bx}$ (BRASIL, 1998), sendo o blend Isabel Precoce x Niágara x BRS Cora o que apresentou menores valores. Resultados parecidos foram encontrados por Haas (2007) trabalhando com blends elaborados com Bordô + Isabel $(70 \%+30 \%)$, Isabel + Bordô $(30 \%+70 \%)$ e Bordô + Concord + Isabel $(30 \%+20 \%+50 \%)$, onde todos os cortes ficaram abaixo do nível estabelecido pela legislação para sólidos solúveis totais, atingindo valores médios de 11,05, 11,85 e 12,19 respectivamente. O blend Niágara x BRS Cora $\times$ BRS Violeta apresentou a maior relação sólidos solúveis/acidez titulável dentre as amostras, atingindo valores médios de 12,91, indicando um maior equilíbrio entre os ácidos e açúcares presentes no suco.

\subsection{VITAMINA C E FENÓLICOS TOTAIS}

O teor de vitamina $\mathrm{C}$ e de fenólicos totais dos diferentes blends de sucos de uva é apresentado na figura 3.

O teor de vitamina $\mathrm{C}$ das amostras diferiu significativamente, sendo o blend Niágara $x$ BRS Cora $x$ BRS Violeta o que apresentou maiores valores médios. Já os blends Niágara x BRS Cora e Isabel Precoce $x$ Niágara x BRS Cora apresentaram valores médios de 27,78 mg.100 mL-1 e 30,19 mg.100 ml-1. Santana et al. (2008), trabalhando com diferentes marcas comerciais de suco de uva integral, encontraram valores que variaram de $16,79 \mathrm{mg} .100 \mathrm{ml}^{-1} \mathrm{a}$ $24,29 \mathrm{mg} .100 \mathrm{ml}^{-1}$, inferiores aos encontrados neste trabalho. 

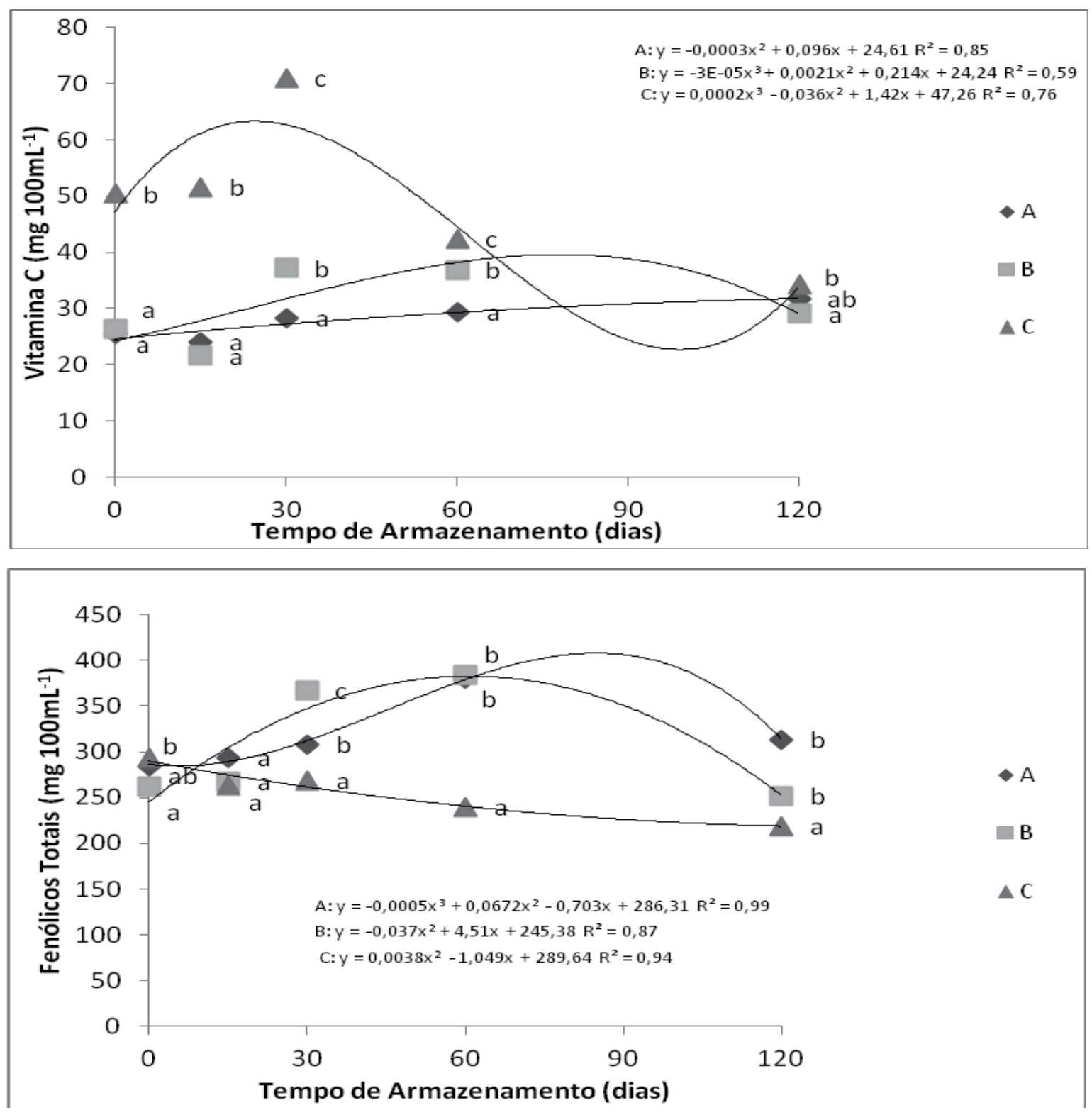

\section{FIGURA 3 - TEOR DE VITAMINA C E DE FENÓLICOS TOTAIS DE DIFERENTES BLENDS DE SUCOS DE UVAS PRODUZIDAS NO SUDOESTE DE MINAS GERAIS (A: NIÁGARA ROSADA X BRS CORA, B: ISABEL PRECOCE X NIÁGARA ROSADA X BRS CORA, C: NIÁGARA ROSADA X BRS CORA X BRS VIOLETA)}

Foi possível observar uma redução do teor de fenólicos totais do blend Niágara $x$ BRS Cora x BRS Violeta ao longo do período de armazenamento, atingindo valores médios de 257,12 mg.100 ml-1. Os demais blends apresentaram aumento no teor de fenólicos e não se diferenciaram entre si. Os compostos fenólicos ou polifenóis constituem um grupo heterogêneo de substâncias encontradas nos alimentos vegetais em altas concentrações, que despertam interesse, sobretudo, pelo potencial antioxidante que apresentam (SCALBERT, WILLIAMSOM, 2000). Assim, os blends de uva apresentaram-se como excelentes fontes de compostos fenólicos, o que está associado com inúmeros benefícios à saúde. Observa-se também uma diferença significativa do teor de fenólicos totais dos blends analisados ao longo do tempo. Segundo Malacrida e Mota (2005), essa variabilidade é justificada por uma série de fatores, que podem interferir diretamente no conteúdo de fenólicos de sucos, como: a cultivar de uva utilizada, o grau de maturação, práticas agrícolas e procedimentos adotados durante a elaboração e o armazenamento dos sucos. 


\subsection{ATIVIDADE ANTIOXIDANTE}

Os antioxidantes podem ser definidos como quaisquer substâncias que, presentes em baixas concentrações, quando comparadas a um substrato oxidável, atrasam ou inibem a oxidação desse substrato de maneira eficaz (SIES e STHAL, 1995; HANDELMAN, 2001). Antolovich et al. (2002) sugerem a realização de mais de um método de análise de antioxidantes, para que cada método contribua para a elucidação de uma parte do complexo fenômeno de inibição da oxidação biológica. Com base nesse pressuposto, podem-se destacar, entre os diversos métodos disponíveis, o sistema de co-oxidação do $\beta$-caroteno/ácido linoleico e o método de sequestro de radicais livres DPPH (2,2-difenil-1-picrilhidrazila), cujas metodologias empregam mecanismos de ação distintos (ARUOMA, 2003; FRANKEL; MEYER, 2000). Dessa forma, a atividade antioxidante dos diferentes blends de sucos de uvas ao longo do armazenamento refrigerado é apresentada na figura 4.
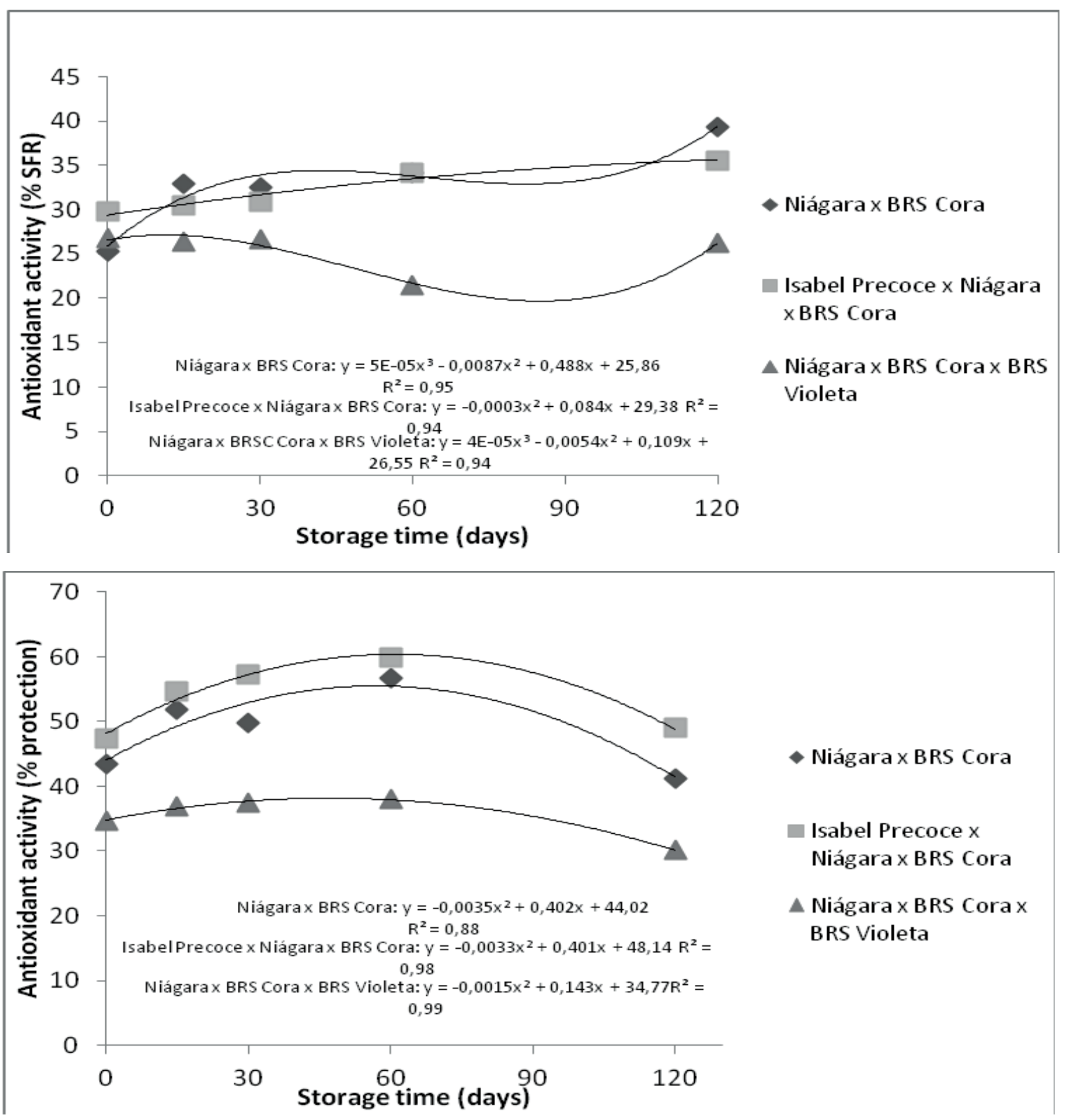

FIGURA 4 - ATIVIDADE ANTIOXIDANTE (\% SRL E \% PROTEÇÃO) DE DIFERENTES BLENDS DE UVAS PRODUZIDAS NO SUDOESTE DE MINAS GERAIS (A: NIÁGARA ROSADA X BRS CORA, B: ISABEL PRECOCE X NIÁGARA ROSADA X BRS CORA, C: NIÁGARA ROSADA X BRS CORA X BRS VIOLETA) 
Dentre as amostras analisadas, observa-se que o blend Niágara x BRS Cora x BRS Violeta apresentou menor porcentagem de sequestro de radicais livres e menor proteção da oxidação do sistema $\beta$-caroteno/ácido linoleico, diferindo significativamente das demais amostras. Muitas pesquisas têm sido realizadas avaliando os efeitos antioxidantes dos compostos fenólicos presentes no vinho (SHAHIDI et al., 1995). Entretanto, alguns autores verificaram, em sucos de uva, atividade antioxidante similar à encontrada em vinhos tintos (FRANKEL et al., 1998; VINSON et al., 1999). O consumo de suco de uva como fonte de compostos fenólicos pode apresentar vantagem em relação ao do vinho, já que a ausência de álcool permite que o suco seja consumido pela maioria das pessoas, inclusive aquelas portadoras de algumas doenças, como, por exemplo, a hepatite, e crianças (ROMERO-PÉREZ et al., 1999).

\section{5 ÁCIDOS ORGÂNICOS}

A tabela 2 apresenta o teor de ácidos orgânicos (ácido málico e ácido tartárico) dos diferentes blends de sucos de uvas produzidas no sudoeste de Minas Gerais.

\section{TABELA 2 - TEOR DE ÁCIDOS ORGÂNICOS (ÁCIDO TARTÁRICO E ÁCIDO MÁLICO) DE DIFERENTES BLENDS DE UVAS PRODUZIDAS NO SUDOESTE DE MINAS GERAIS}

\begin{tabular}{ccc}
\hline Blends & Ácido tartárico $\left(\mathbf{g ~ L}^{-1}\right)$ & Ácido málico $\left(\mathbf{g ~ L}^{-1}\right)$ \\
\hline Niágara $\times$ BRS Cora & $2,58 \mathrm{ab}$ & $3,01 \mathrm{~b}$ \\
\hline Isabel Precoce $\times$ Niágara $\times$ BRS Cora & $2,82 \mathrm{~b}$ & $3,75 \mathrm{~b}$ \\
\hline Niágara x BRS Cora x BRS Violeta & $2,03 \mathrm{a}$ & $1,74 \mathrm{a}$ \\
\hline
\end{tabular}

Letras minúsculas indicam diferença entre os tratamentos ao nível de $5 \%$ de significância.

O teor de ácidos orgânicos (ácido málico e ácido tartárico) mostrou diferenças significativas entre os diferentes blends, mas não apresentou diferenças significativas ao longo do tempo de armazenamento. Sendo assim, quanto ao teor de ácido tartárico, o blend Isabel Precoce x Niágara x BRS Cora não se diferenciou do blend Niágara x BRS Cora, e este, por sua vez, não se diferenciou do blend Niágara x BRS Cora x BRS Violeta. Com relação ao teor de ácido málico, os blends Niágara x BRS Cora e Isabel Precoce x Niágara x BRS Cora não apresentam diferenças entre si, porém se diferenciaram estatisticamente do blend Niágara $\mathrm{x}$ BRS Cora x BRS Violeta, que apresentou menores valores. Soyer et al. (2003), avaliando o teor de ácidos orgânicos de 11 sucos de uva produzidos na Turquia, observaram teores de ácido málico que variaram de $1,36 \mathrm{~g} \mathrm{~L}^{-1}$ a $3,47 \mathrm{~g} \mathrm{~L}^{-1}$. Resultados parecidos foram encontrados no presente estudo, em que os teores obtidos para os diferentes blends variaram de $1,74 \mathrm{~g} \mathrm{~L}^{-1}$ a $3,47 \mathrm{~g} \mathrm{~L}^{-1}$.

\section{CONCLUSÃO}

Blends de sucos de uvas produzidas no sudoeste de Minas Gerais constituem uma excelente alternativa de produto a ser elaborado, por serem excelentes fontes de compostos fenólicos e 
vitamina C. O blend Niágara x BRS Cora x BRS Violeta apresentou maior teor de antocianinas e vitamina $C$, menor acidez e maior relação sólidos solúveis/acidez titulável, indicando um maior equilíbrio entre os sabores doce e ácido.

\section{ABSTRAT}

\section{ANTIOXIDANT ACTIVITY AND TOTAL PHENOLICS IN BLENDS OF JUICES FROM AMERICAN GRAPES PRODUCED IN THE SOUTHWEST OF MINAS GERAIS}

The aim of this study was characterize and evaluate the antioxidant activity of juices from American grapes produced in the Southwest of Minas Gerais. The fruit were harvested, selected and sanitized and blends from Niagara Rosada $x$ BRS Cora $(50 \%+50 \%)$, Niagara Rosada $x$ BRS Cora x BRS Violeta $(40 \%+30 \%+30 \%)$ and Isabel Precoce $x$ Niagara Rosada x BRS Cora $(25 \%+25 \%+50 \%)$ were produced, and stored at $18{ }^{\circ} \mathrm{C} \pm 2{ }^{\circ} \mathrm{C}$ for 120 days. The following analysis were performed during storage: color $\left(\mathrm{L}^{*}\right), \mathrm{pH}$, soluble solids, titratable acidity, soluble solids/titratable acidity ratio, total anthocyanins, antioxidant activity (DPPH and $\beta$-carotene/ linoleic acid), total phenolics, vitamin $c$ and organic acids. The blend Niagara Rosada $x$ BRS Cora x BRS Violeta showed higher total anthocyanins and vitamin c levels, lower acidity and higher soluble solids/ acidity ratio, indicating a desirable balance between sweet and sour flavors.

KEY-WORDS: GRAPE JUICE; ANTHOCYANINS; VITAMIN C; ORGANIC ACIDS; QUALITY.

\section{REFERÊNCIAS}

1 ANDRADE, P. B., MENDES, G., FALCO, V., VALENTÃO, P., SEABRA, R. M. Preliminary study of flavonols in port wine grape varieties. Food Chemistry, v. 73, p. 397-399, 2001.

2 ANTOLOVICH, M., PRENZlER, P. D., PATSALIDES, E., MCDONALD, S., ROBARDS, K. Methods for testing antioxidant activity, Analyst, v. 127, p. 183-198, 2002.

3 ARUOMA, O.I. Methodological characterizations for characterizing potential antioxidant actions of bioactive components in plant foods, Mutation Research, v. 9, p. 523-524, 2003.

4 ASSOCIATION OF OFFICIAL ANALYTICAL CHEMISTS. Official Methods of the Association of the Agricultural Chemists, eighteen ed. Gaithersburg: Association of Official Analytical Chemists, 2007.

5 BORGES, R. S., PRUDÊNCIO, S. H., ROBERTO, S. R., ASSIS, A. M. Avaliação sensorial de suco de uva cv Isabel em cortes com diferentes cultivares. Revista Brasileira de Fruticultura, volume especial, p. 584-591, 2011.

6 BRASIL. Ministério da agricultura. Portaria N. 544 de 16 de novembro de 1998. Aprova o regulamento técnico para fixação dos padrões de identidade e qualidade para refresco. Diário oficial [da] república federativa do brasil, Brasília, 10 Nov. 1998. Disponível em: <http://oc4j.agricultura.gov.br/ agrolegis/do/consultalei ?op = viewtextual\&codigo=1150>. Acesso em: 30 Abr. 2010.

7 CHEDEA, V. S., BRAICU, C.; SOCACIU, C. Antioxidant/prooxidant activity of a poliphenolic grape seed extract. Food Chemistry, v. 121, p. 132-139, 2010.

8 CHITARRA, M. I. F.; CHITARRA, A. B. Pós-colheita de frutos e hortaliças: fisiologia e manuseio. 2. ed. Lavras: UFLA, 2005.

9 COMISSION INTERNATIONALE DE I'ECLAIRAGE. Recommendations on uniform color spaces-color difference equations, psychomettic color terms. Paris: CIE, 1978.

10 DUARTE-ALMEIDA, J. M., SANTOS, R. J., GENOVESE, M. I., LAJOLO, F. M. Avaliação da atividade antioxidante utilizando sistema $\beta$-caroteno/ácido linoléico e método de seqüestro de radicais DPPH. Ciência e Tecnologia de Alimentos, v. 26, p. 446-452, 2006. 
11 FRANCIA-ARICHA, F. M., GUERRA, M. T., RIVAS-GONZALO, I. C., SANTOS-BUELGA, C. New anthocyanin pigments formed after condensation with flavonols. Journal of Agricultural and Food Chemistry, v. 45, p. 2262-2266, 1997.

12 FRANKEL, E. N., BOSANEK, C. A., MEYER, A. S., SILLIMAN, K., KIRK, L. L. Commercial grape juice inhibits the in vitro oxidation of human low-density lipoproteins. Journal of Agricultural and Food Chemistry, v. 46, p. 834-838, 1998.

13 FRANKEL, E. N.; MEYER, A. S. The problems of using one-dimensional methods to evaluate multifunctional food and biology antioxidants. Journal of Science of Food and Agriculture, v. 80, p. 1925-1941, 2000.

14 GARDNER, P. T., WHITE, T. A. C.; MCPHAIL, D. B., DUTHIE, G. G. The relative contributions of vitamin C, carotenoids and phenolics to the antioxidant potential of fruit juices. Food Chemistry, v. 68, p. 471-474, 2000.

15 GENOVA, G., IACOPINI, P., BALDI, M., RANIERI, A., STORCHI, P., SEBASTIANI, L. Temperature and storage effects on antioxidant activity of juice from red and white grapes. International Journal of Food Science and Technology, v. 47, p. 13-23, 2012.

16 GIUSTI, M. M.; WROLSTAD, R. E. Anthocyanins: characterization and measurement with uv-visible spectroscopy. In: WROLSTAD, R.E. Current protocols in food analytical chemistry, cap. 1, p. 1-13, 2001.

17 GURAK, P. D., CABRAL, L.M.C., ROCHA-LEÃO, M.H.M., MATTA, V.M., FREITAS, S.P . Quality evaluation of grape juice concentrated by reverse osmosis. Journal of Food Engineering, v. 96, p. 421-426, 2010.

18 HAAS, L. I. R. Caracterização e estudo de compostos em sucos e blends de uvas americanas produzidas em Pelotas-RS. 2007. 97f. Dissertação (Mestrado em Ciência e Tecnologia Agroindustrial) - Universidade Federal de Pelotas, 2007.

19 HANDELMAN, G. J. The evolving role of carotenoids in human biochemistry. Nutrition, v. 17, p. 818-822, 2001.

20 LARRAURI, J. A.; RUPÉREZ, P.; SAURA-CALIXTO, F. Effect of drying temperature on the stabilitity of polyphenols and antioxidant activity of red grape pomace peels. Journal of Agricultural and Food Chemistry, v. 45, p. 1390-1393, 1997.

21 LUCENA, A. P. S., NASCIMENTO, R. J. B., MACIEL, J. A. C., TAVARES, J. X., BARBOSA-FILHO, J., OLIVEIRA, E. J. Antioxidant activity and phenolics content of selected Brazilian wines. Journal of Food Composition and Analysis, San Diego, v. 23, p. 30-36, 2010.

22 MALACRIDA, C. R.; MOTTA, S. Compostos fenólicos totais e antocianinas em suco de uva. Ciência e Tecnologia de Alimentos, v. 25, p. 659-664, 2005.

23 RIZZON, L.A., MANFROI, V.; MENEGUZZO, J. Elaboração de suco de uva na propriedade vitícola, Bento Gonçalves: EMBRAPA Uva e Vinho, 1998.

24 RIZZON, L. A., MIELE, A.; MENEGUZZO, J. Avaliação da uva cv Isabel para a elaboração de vinho tinto. Ciência e Tecnologia de Alimentos, v. 20, p. 115-121, 2000.

25 ROMERO-PÉREZ, A. I., IBERN-GÓMES, M., LAMUELA-RAVENTÓS, R. M., TORRE-BORONAT, M. C. Piceid, the major resveratrol derivative in grape juice. Journal of Agricultural and Food Chemistry, v. 47, p. 1533-1536, 1999.

26 RUFINO, M. S. M., ALVES, R. E., BRITO, E.S., MORAIS, S. M., SAMPAIO, C. G., JIMENEZ, J. P, SAURACALIXTO, F. D. Metodologia científica: determinação da atividade antioxidante total em frutas pela captura do radical livre DPPH. Fortaleza: EMBRAPA, 2007a 4p. (Comunicado técnico, 127).

27 RUFINO, M. S. M. ALVES, R. E.; BRITO, E. S.; FIHO, J. M., MOREIRA, A. V. B. Metodologia científica: determinação da atividade antioxidante total em frutas no sistema $\beta$ caroteno - ácido linoléico. Fortaleza: EMBRAPA, 2007b. 4p. (Comunicado técnico, 126).

28 SANTANA, M. T. A., SIQUEIRA, HH, REIS, KC, LIMA, LCO AND SILVA, RJL. Caracterização de diferentes marcas de sucos de uva comercializados em duas regiões do Brasil. Ciência e Agrotecnologia, v. 32, p. 882-886, 2008.

29 SCALBERT, A., WILLIAMSON, G. Dietary intake and bioavailability of polyphenols. Journal of Nutririon, v. 130, p. 2073-2085, 2000.

30 SHAHIDI, F., NACZK, M. Food phenolics: sources, chemistry, effects and applications. Lancaster: Technomic, 1995, $331 \mathrm{p}$.

31 SIES, H., STHAL, W. Vitamins E and C, $\beta$-carotene and other carotenoids as antioxidants. The American Journal of Clinical Nutrition, v. 62, p. 1315-1321, 1995. 
32 SINGLETON, V. L.; ROSSI, J. A. Colorimetry of total phenolics with phosphomolybdic-phosphotungstic acid reagents. American Journal of Enology and Viticulture, v. 16, p. 144-158, 1965.

33 SOYER, Y.; KOCA, N.; KARADENIZ, F. Organic acid profile of Turkish white grapes and grape juices. Journal of Food Composition and Analysis, San Diego, v. 16, n. 5, p. 629-636, oct. 2003.

34 STROECKHER, R.; HENNING, H. M. Analisis de vitaminas: metodos comprobados. Madrid: Paz Montalvo, 428p, 1967.

35 VINSON, J. A., JANG, J, YANG, J, DABBAGH, Y, LIANG, X, SERRY, M, PROCH, J AND CAI, S. Vitamins and especially flavonoids in common beverages are powerful in vitro antioxidants which enrich lower density lipoproteins and increase their oxidative resistance after ex vivo spiking in human plasma. Journal of Agricultural and Food Chemistry, v. 47, p. 2502-2504, 1999. 